\title{
The Cost-Utility of Intravenous Magnesium Sulfate for Treating Acute Asthma in Children
}

\author{
jefferson buendia ${ }^{1}$, Ranniery Acuña-Cordero ${ }^{2}$, and Carlos Rodriguez-Martinez ${ }^{3}$ \\ ${ }^{1}$ University of Antioquia \\ ${ }^{2}$ Hospital Militar Central \\ ${ }^{3}$ School of Medicine, Universidad El Bosque
}

May 13, 2020

\begin{abstract}
Introduction Despite the evidence supporting the use of intravenous Magnesium Sulfate (MS) in acute asthma; this drug continues being considered as the second line in pediatric acute asthma exacerbations. This study aimed to evaluate the cost-effectiveness of the MS in acute asthma. Methods A decision tree model was used to estimate the Cost-utility study that compared MS versus standard treatment (control group) in an infant with acute asthma in the emergency setting. Cost data were obtained from a retrospective study on asthma from tertiary centers in Rionegro, Colombia, while utilities were collected from the literature. The analysis was carried out from a societal perspective. Results The model showed that MS for treating pediatric patient with acute asthma, was associated with lower total cost than standard therapy (US $\$ 1149$ vs US $\$ 1598$ average cost per patient), and higher QALYs ( 0.60 vs 0.52 average per patient); showing dominance. the probabilty that MS provides a more cost-effective use of resources compared with standard therapy exceeds $99 \%$ for all willingness to pay thresholds Conclusion MS in emergency settings was cost-effective for the hospital treatment of an infant with asthma moderate or severe. Our study provides evidence that should be used by decision-makers to improve clinical practice guidelines and should be replicated to validate their results in other middle-income countries.
\end{abstract}

\section{The Cost-Utility of Intravenous Magnesium Sulfate for Treating Acute Asthma in Children} Short running title: Intravenous Magnesium Sulfate in Asthma

Jefferson Antonio Buendía, MD,MSc, $\mathrm{PhD}^{1}$, Ranniery Acuña-Cordero $\mathrm{MD}^{2}$, Carlos E. Rodriguez-Martinez $\mathrm{MD}, \mathrm{MSc}, \mathrm{PhD}(\mathrm{c})^{3,4}$

1. Department of Pharmacology and Toxicology, School of Medicine, Research group in pharmacology and toxicology (INFARTO). Universidad de Antioquia, Medellín, Colombia.

2. Departamento de Neumología Pediátrica, Hospital Militar Central, Departamento de Pediatría, Facultad de Medicina, Universidad Militar Nueva Granada, Bogotá, Colombia

3. Department of Pediatrics, School of Medicine, Universidad Nacional de Colombia, Bogota, Colombia

4. Department of Pediatric Pulmonology and Pediatric Critical Care Medicine, School of Medicine, Universidad El Bosque, Bogota, Colombia

Corresponding author: Jefferson A. Buendía, MD, Ph.D., Facultad de Medicina, Universidad de Antioquia, Carrera 51D \#62-29, Tel: +574 2196046; Medellín, Colombia. E-mail: jefferson.buendia@gmail.com. E-mail: JAB: jefferson.buendia@gmail.com,

\section{Introduction}

Despite the evidence supporting the use of intravenous Magnesium Sulfate (MS) in acute asthma; this drug 
continues being considered as the second line in pediatric acute asthma exacerbations. This study aimed to evaluate the cost-effectiveness of the MS in acute asthma.

\section{Methods}

A decision tree model was used to estimate the Cost-utility study that compared MS versus standard treatment (control group) in an infant with acute asthma in the emergency setting. Cost data were obtained from a retrospective study on asthma from tertiary centers in Rionegro, Colombia, while utilities were collected from the literature. The analysis was carried out from a societal perspective.

\section{Results}

The model showed that MS for treating pediatric patient with acute asthma, was associated with lower total cost than standard therapy (US $\$ 1149$ vs US $\$ 1598$ average cost per patient), and higher QALYs ( 0.60 vs 0.52 average per patient); showing dominance. the probabilty that MS provides a more cost-effective use of resources compared with standard therapy exceeds $99 \%$ for all willingness to pay thresholds

\section{Conclusion}

MS in emergency settings was cost-effective for the hospital treatment of an infant with asthma moderate or severe. Our study provides evidence that should be used by decision-makers to improve clinical practice guidelines and should be replicated to validate their results in other middle-income countries.

Keywords: health economics, public health, healthcare

\section{Introduction}

The magnesium sulfate (MS) is a medication with bronchodilator properties described for more than 50 years (1) .However, this medication have not been considered in the first-line drug in acute asthma, - in part probably due to the limited number and size of studies (2) in the last century - and their use, now, is more extensive than current guidelines or available evidence appear to support (3)

Growing evidence shows significant improvement in pulmonary function and a reduction of hospital admissions in children (4). The positive effect, in patients with asthma, is mediated through its role as a calcium antagonist, as an enzymatic cofactor of sodium and potassium flux across cell membranes (5) . Theses physiological effects has been reflected in randomized clinical trials in children, reducing asthma severity scores, especially in severe asthma, with no differences in the occurrence of adverse events respect to standard inhaled treatment (6).

Despite this evidence and the frequent use of MS in acute asthma; this drug continues being considered as the second line in pediatric acute asthma exacerbations. A recent meta-analysis has demonstrated their benefit of early use in a patient who did not respond after of rescue within 1-2 hrs to b2-agonist without serious adverse events (7) (8). The economic impact of these benefits has been not evaluated yet, and obtain this evidence could be important as a prior step to adopt this drug in as first line in the clinical guidelines of asthma. Just their early use in the emergency setting is where this drug could have more economic impact, reducing cost and morbidity associated with treatment failure and mechanical ventilation. This will be more important especially in many hospitals of middle-income countries that have a low frequency of PICU services per habitant, and where this drug can be a cost-saving alternative. This study aims to evaluate the cost-effectiveness of MS in an infant with acute asthma in the emergency setting in Colombia.

\section{Methods}

\section{Design}

Cost-utility study that compared intravenous MS versus standard treatment (control group) in an infant with acute asthma in the emergency setting. The effectiveness outcome was the quality-adjusted life years (QALYs). The analysis was carried out from a societal perspective (included direct and indirect costs). The analytic horizon was an acute episode of asthma. Given the short time horizon, no type of discount to costs 
or results was applied. The study protocol was reviewed and approved by the Institutional Review Board of Clinica Somer (No 281015) and the University of Antioquia (No 18/2015).

\section{Decision Analytic Model}

A decision tree model was used to estimate the cost-effectiveness of the MS in acute asthma (Figure 1 ). We defined the following outcomes according to the natural history of acute asthma: home treatment, hospitalization, need of mechanical ventilation support and death.

\section{Probabilities}

To estimate the probabilities of the model (see Table 1 ), we performed a systematic review of systematic reviews or RCTs published or observational studies up to January, 2020. In order to identify potentially relevant studies were made searches of computerized databases (MEDLINE, CENTRAL, LILACS, and CINAHL) ; using the following search strategy: (Magnesium Sulfate OR Sulfate, Magnesium OR Magnesium Sulfate, Heptahydrate OR Heptahydrate Magnesium Sulfate) AND ((asthma) AND (acute exacerbation) . No language restrictions were applied. To be included in the model, the studies had to be RCTs of parallelgroup or cross-over design, systematic reviews or RCTs published or observational studies include children between 2 and 18 years of age. Also were included others observational clinical obtained during the review of references cited in published literature identified the potentially applicable studies. The computerized search yield 49 citations, and a total of 32 studies were examined in full text for possible inclusion. For inclusion of data in the model, participants in the studies needed to be under the age of 18 years with acute exacerbation asthma, studies needed to evaluated the use of MS and report at least one of the following outcomes: percentage of hospitalizacions or admission in pediatric intensive unit or acute complications during the period of observation. Studies were excluded if they included patients with no clear diagnosis of asthma and patients over 18 years. All probabilities were contrasted with probabilities extracted from local studies to derivate the ranges in the sensibility analisis (8-16).

\section{Intervention}

Information regarding the effect of MS was extracted from a recent systematic review and meta-analysis included in the review mentioned previously(8). This study included trials that evaluate the effect of MS ( $25-50 \mathrm{mg} / \mathrm{kg}$ through $20 \mathrm{~min}$ ) in patients (range of age of 6-18 years) with acute asthma moderate-severe. All children were treated initially with inhaled albuterol $(0.15 \mathrm{mg} / \mathrm{kg})$ by facemask or mouthpiece from nebulizer. On completion of this treatment and after of confirmation a peak expiratory flow rate less than $60 \%$, in all children were given a second albuterol treatment, intravenous methylprednisolone, and intravenous magnesium sulfate or placebo (normal saline solution)(17-19) .

\section{Cost analysis:}

We included infants under two years of age admitted in tertiary centers, in Rionegro, Colombia due to asthma according to the national clinical guideline of asthma in children from January 2018 to December 2018. Our country has been characterized by having a very small price variation in the last 10 years, especially in health services between the different clinics and over time (20). Moreover, the proportions of each of the costs remain relatively constant, which, with few variations in their composition in the last ten years (20). The municipality of Rionegro had a total population of 101,046 inhabitants, and hast two tertiary referral hospital (21) All cost and use of resources were collected directly from medical invoices and electronic medical records. The direct costs considered in the analysis include medical consultation at emergency room, specialist referrals, chest physiotherapy, diagnosis support (laboratory, electrocardiogram, x-ray, etc), medication (oxygen, nebulization, antibiotics, corticosteroids, bronchodilators, etc.), medical devices, hotel services at intensive care unit, hotel services in general medical ward, table 2 .

We use US dollars (Currency rate: US $\$ 1.00=\operatorname{COP} \$ 3,000)(22)$ to express all costs in the study. For the valuation of the indirect costs associated with the loss of productivity of parents, the human capital method was used, assuming everyone receives an income of at least a legal minimum wage for formal or informal work. The cost-opportunity of the productivity loss at the workplace and the caregiver was assessed based on 
the minimum wage without including the transportation assistance for the year 2019 (U\$229.81 per month). The legal minimum wage approved by the government was taken as a reference and not an average or median wage thereof, given that in Colombia more than $75 \%$ of the population has this value as income(23). Because all patients with acute ashtma eísode included were children, we assumed that at least one family, member accompanies the patient permanently during hospitalization, since pediatric hospitals in the country usually allow only one companion per patient in the hospital. The cost associated with transportation and food (does not include a stay), was assumed to correspond to $50 \%$ of minimum wage per day.

\section{Utilities}

The utility values used to QALY calculations were collected from the literature. The utility value for no hospitalization or mild symptoms was 0.75 , for hospitalization or moderate symptoms 0,52 and for need of mechanical ventilation 0,24 (24). Given that they are utilities extracted from studies in populations other than the Colombian, a range was used for the sensitivity analysis of one way and probabilistic of more or less $20 \%$ the value of the utility.

\section{Sensitivity analyses}

The robustness was evaluated with one-way sensitivity analyses (SA) of base case parameters with the greatest level of uncertainty such as utilities, hospital-related costs, and the probability of hospitalization. Tornado diagrams were used as graphical method for displaying one-way sensitivity analyses. Probabilistic sensitivity analysis was made using the Monte Carlo technique with a simulation of a hypothetical cohort of 10000 patients in which each parameter varied randomly according to certain distributions (beta distribution in the case of probabilities, and gamma distribution in the case of costs); to generate expected cost utilities with a $95 \%$ confidence intervals $(95 \% \mathrm{CI})$. To evaluate the uncertainty surrounding the cost-effectiveness of MS a cost-effectiveness acceptability curve was used. We estimated the population expected value of perfect information to inform the expected cost of uncertainty (expected opportunity loss surrounding the decision) (25). Microsoft Exel @ $\cap$ was used in all analyzes.

\section{Results}

The model showed that MS for treating pediatric patient with acute asthma, was associated with lower total cost than standard therapy (US $\$ 1149$ vs US $\$ 1598$ average cost per patient), and higher QALYs ( 0.60 vs 0.52 average per patient); showing dominance. A position of dominance negates the need to calculate an incremental cost-effectiveness ratio (Table 3 ).

\section{Sensitivity analyses}

One-way and tornado diagram showed that the probability of hospitalization has the highest impact on the outcome, but MS was the dominant strategy in all range of probabilities analyzed. The results of PSA are graphically represented in the cost-effectiveness plane,figure $2.70 .87 \%$ of simulations were graphed in quadrant 2 (lower cost, high QALYs), and $28.4 \%$ in quadrant 1 of this plane (high cost, high QALYs) . The $95 \%$ CI for the cost per patient treated with SM and with standard treatment were US\$1042 to 1257 , and US\$ 1471 to 1725 . The $95 \%$ CI for QALYs per patient was 0.59 to 0.62 , and 0.51 to 0.54 respectively. The Cost-effectiveness acceptability curve shows that the probabilty that MS provides a more cost-effective use of resources compared with standard therapy exceeds $99 \%$ for all willingness to pay thresholds. The population expected value of perfect information (EVPIP) for threshold of US $\$ 20000$ (threshold of 3 times the Colombian per capita gross domestic product) was US\$221517 which was 15 times less than maximum EVPIP simulated by the model.

\section{Discussion}

Our study suggests that intravenous MS are unequivocally cost-effective and dominant over standard treatment in children with moderate-severe acute asthma in emergency setting; achieving better outcomes at a lower cost. Although the variable that exhibited a significant effect on these results was the probability of hospitalization, MS was the dominant strategy overall ranges of this variable analyzed. 
The findings of this study constitute a new argument to include the MS in clinical practice guidelines of pediatric asthma for treating acute asthma exacerbations. Due to the important burden of disease generated by asthma, especially in Colombia and other middle-income countries, this strategy will be useful not only for decreasing the probability of hospitalization due to acute asthma but also for decreasing associated costs. The potential magnitude of cost savings for the health system (US $\$ 448$ per patient ) is no negligible if we consider that this disease affects between 10 to $13 \%$ of children in Colombia and only $2.4 \%$ of them meets criteria for total asthma control $(26,27)$

The last version of Global Initiative for Asthma does no recommend the use of MS for routine use in children with acute exacerbation, despite acknowledging that there is evidence of its use and positive effects on hospitalization rates (28). Also the Colombian Clinical Guideline for Asthma do not recognize the MS as first line in this patients(29). Our study provides evidence of beneficial clinical effects of MS when added to the standard treatment with bronchodilators, and corticosteroids in patient with moderate-severe asthma exacerbations.

Previous economic evaluation in the UK based on evidence from a randomized placebo-controlled multi-center trial of nebulized MgSO4 in severe acute asthma in children found that MS had a $67.6 \%$ of probability of being cost-effective at EUR 22.957 per QALY gained(30). However, they only had full costing in less than $50 \%$ of cases, limiting their conclusions. Our study is the second publication on this topic, using a societal perspective, and made in a setting with it more limited economic resources. The results from different countries are not easily comparable, mainly due to many differences in prices of labor, drugs, medical test, frequency of use of resources, adherence to clinical guidelines, and differences in access to and provision of specialized care instead of primary care, and payment schemes.

A very important aspect of our model is that it was robust to changing the values of the model's utilities and costs. MS was always the cost-effectiveness strategy un all ranges of thresholds evaluated with a low population EVPI. This was consistent with the finding that although that our utilities were collected from the other population, our results did not change when exploring the change in the ICER in the range of values of each utility explored. The same happens with costs. Although the resources, frequencies of use, and costs were collected from tertiary centers, in Rionegro, and not from a national study with all hospitals in Colombia, modifications to their values in the sensitivity analysis also did not significantly change the ICER. These aspects give us confidence regarding the ability to make decisions with our results; as is always necessary for science, more studies to replicate our results $(31,32)$. Our study has some limitations. The cost data were collected retrospectively. Asthma treatment and the costs in question, including hospital prices, did not markedly change to our days. Furthermore, our country has been characterized by having a very small price variation in the last 10 years, especially in health services (20). Additionally, we use utilities extracted from the literature and not estimated directly from our population. As was mentioned previously, the reliability and robustness of the results were evaluated by sensitivity analyses.

In conclusion, the MS in emergency settings was cost-effective for the hospital treatment of an infant with asthma moderate or severe. Our study provides evidence that should be used by decision-makers to improve clinical practice guidelines and should be replicated to validate their results in other middle-income countries.

\section{References}

1. VG H. The bronchodilator action of magnesium and its antagonistic action (dilator action) against pilocarpine, histamine and barium chloride. . J Pharmacol Exp Ther. 1938;64.

2. Stenberg RG, Winniford MD, Hillis LD, Dowling GP, Buja LM. Simultaneous acute thrombosis of two major coronary arteries following intravenous cocaine use. Arch Pathol Lab Med. 1989;113(5):521-4.

3. Jones LA, Goodacre S. Magnesium sulphate in the treatment of acute asthma: evaluation of current practice in adult emergency departments. Emerg Med J. 2009;26(11):783-5.

4. Shan Z, Rong Y, Yang W, Wang D, Yao P, Xie J, et al. Intravenous and nebulized magnesium sulfate for treating acute asthma in adults and children: a systematic review and meta-analysis. Respir Med. 
2013;107(3):321-30.

5. D'Angelo EK, Singer HA, Rembold CM. Magnesium relaxes arterial smooth muscle by decreasing intracellular Ca2+ without changing intracellular Mg2+. J Clin Invest. 1992;89(6):1988-94.

6. Powell C, Kolamunnage-Dona R, Lowe J, Boland A, Petrou S, Doull I, et al. Magnesium sulphate in acute severe asthma in children (MAGNETIC): a randomised, placebo-controlled trial. Lancet Respir Med. 2013;1(4):301-8.

7. Irazuzta JE, Chiriboga N. Magnesium sulfate infusion for acute asthma in the emergency department. J Pediatr (Rio J). 2017;93 Suppl 1:19-25.

8. Su Z, Li R, Gai Z. Intravenous and Nebulized Magnesium Sulfate for Treating Acute Asthma in Children: A Systematic Review and Meta-Analysis. Pediatr Emerg Care. 2018;34(6):390-5.

9. Vergara C, Caraballo L. Asthma mortality in Columbia. Ann Allergy Asthma Immunol. 1998;80(1):55-60.

10. Forno E, Gogna M, Cepeda A, Yanez A, Sole D, Cooper P, et al. Asthma in Latin America. Thorax. 2015;70(9):898-905.

11. Rodriguez Martinez CE, Guzman MC, Castillo JM, Sossa MP, Ojeda P. Evaluation of clinical criteria for the acute respiratory distress syndrome in pediatric patients. Pediatr Crit Care Med. 2006;7(4):335-9.

12. Dennis RJ, Caraballo L, Garcia E, Rojas MX, Rondon MA, Perez A, et al. Prevalence of asthma and other allergic conditions in Colombia 2009-2010: a cross-sectional study. BMC Pulm Med. 2012;12:17.

13. Rodriguez-Martinez CE, Sossa-Briceno MP, Castro-Rodriguez JA. Metered-dose inhalers vs nebulization for the delivery of albuterol in pediatric asthma exacerbations: A cost-effectiveness analysis in a middleincome country. Pediatr Pulmonol. 2020;55(4):866-73.

14. Neffen H, Moraes F, Viana K, Di Boscio V, Levy G, Vieira C, et al. Asthma severity in four countries of Latin America. BMC Pulm Med. 2019;19(1):123.

15. Rodriguez-Martinez CE, Sossa-Briceno MP, Castro-Rodriguez JA. Predictors of hospitalization for asthma in children: results of a 1-year prospective study. Pediatr Pulmonol. 2014;49(11):1058-64.

16. Rampersad N, Wilkins B, Egan JR. Outcomes of paediatric critical care asthma patients. J Paediatr Child Health. 2018;54(6):633-7.

17. Scarfone RJ, Loiselle JM, Joffe MD, Mull CC, Stiller S, Thompson K, et al. A randomized trial of magnesium in the emergency department treatment of children with asthma. Ann Emerg Med. 2000;36(6):572-8.

18. Ciarallo L, Sauer AH, Shannon MW. Intravenous magnesium therapy for moderate to severe pediatric asthma: results of a randomized, placebo-controlled trial. J Pediatr. 1996;129(6):809-14.

19. Ciarallo L, Brousseau D, Reinert S. Higher-dose intravenous magnesium therapy for children with moderate to severe acute asthma. Arch Pediatr Adolesc Med. 2000;154(10):979-83.

20. Estadisticas DAN. Indice de Precios al Consumidor - IPC 2020 [Available from:https://www.dane.gov.co/index.php/estadisticas-por-tema/precios-y-costos/indice-de-precios-alconsumidor-ipc

21. Estadisticas DAN. Proyecciones de poblacion 2018 [03/07/2019]. Available from:https://www.dane.gov.co/index.php/estadisticas-por-tema/demografia-y-poblacion/proyecciones-depoblacion.

22. la Bd, Republica. Tasa Representativa del Mercado (TRM - Peso por dolar) 2019 [cited 2020. Available from:https://www.banrep.gov.co/es/estadisticas/trm.

23. Departamento, Nacional, (DANE) DE. Archivo Nacional de Datos 2019 [Available from:https://sitios.dane.gov.co/anda-index/. 
24. Chiou CF, Weaver MR, Bell MA, Lee TA, Krieger JW. Development of the multi-attribute Pediatric Asthma Health Outcome Measure (PAHOM). Int J Qual Health Care. 2005;17(1):23-30.

25. Felli JC, Hazen GB. Sensitivity analysis and the expected value of perfect information. Med Decis Making. 1998;18(1):95-109.

26. Neffen H, Fritscher C, Schacht FC, Levy G, Chiarella P, Soriano JB, et al. Asthma control in Latin America: the Asthma Insights and Reality in Latin America (AIRLA) survey. Rev Panam Salud Publica. $2005 ; 17(3): 191-7$.

27. Garcia E, Aristizabal G, Vasquez C, Rodriguez-Martinez CE, Sarmiento OL, Satizabal CL. Prevalence of and factors associated with current asthma symptoms in school children aged 6-7 and 13-14 yr old in Bogota, Colombia. Pediatr Allergy Immunol. 2008;19(4):307-14.

28. Global Iniciative for Asthma Management and Prevention 2020 [Available from:www.ginaasthma.org.

29. Colciencias MdSyPS-. Guia de Practica Clinica Para el diagnostico, atencion integral y seguimiento de ninos y ninas con diagnostico de Asma. Guia No. 012014 [Available from: ,http://gpc.minsalud.gov.co/gpc-sites/Repositorio/Conv_500/GPC_asma/GPC_Comp_Asma.pdf.

30. Petrou S, Boland A, Khan K, Powell C, Kolamunnage-Dona R, Lowe J, et al. Economic evaluation of nebulized magnesium sulphate in acute severe asthma in children. Int J Technol Assess Health Care. 2014;30(4):354-60.

31. Buendia JA. [Attitudes, knowledge and beliefs of patient about anti-hypertensive drugs]. Biomedica. 2012;32(4):578-84.

32. Buendia JA, Otamendi E, Kravetz MC, Cairo F, Ruf A, de Davila M, et al. Combinational Effect of CYP3A5 and MDR-1 Polymorphisms on Tacrolimus Pharmacokinetics in Liver Transplant Patients. Exp Clin Transplant. 2015;13(5):441-8.

33. Rodriguez-Martinez CE, Sossa-Briceno MP, Castro-Rodriguez JA. Advantage of inhaled corticosteroids as additional therapy to systemic corticosteroids for pediatric acute asthma exacerbations: a costeffectiveness analysis. J Asthma. 2019:1-10.

\section{Acknowledgements : none}

Conflict of interest statement for all authors : All authors declare that they do not have any conflict of interest in this publication.

\section{Figure legends}

Figure 1. Decision tree model with probabilities estimated by outcome

Figure 2. Cost effectiveness plane

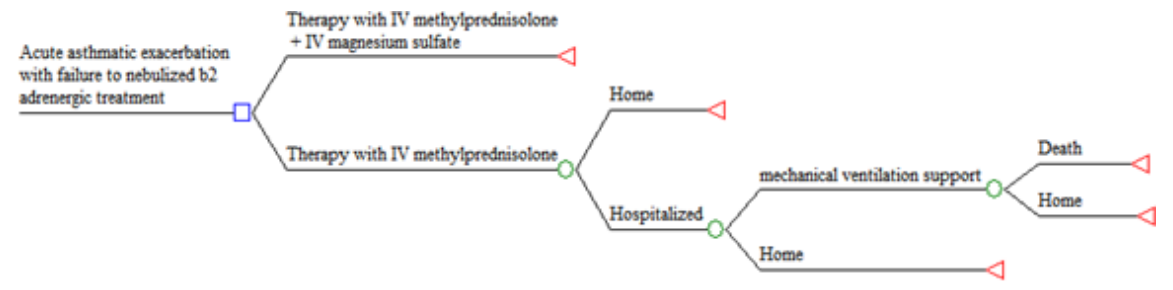




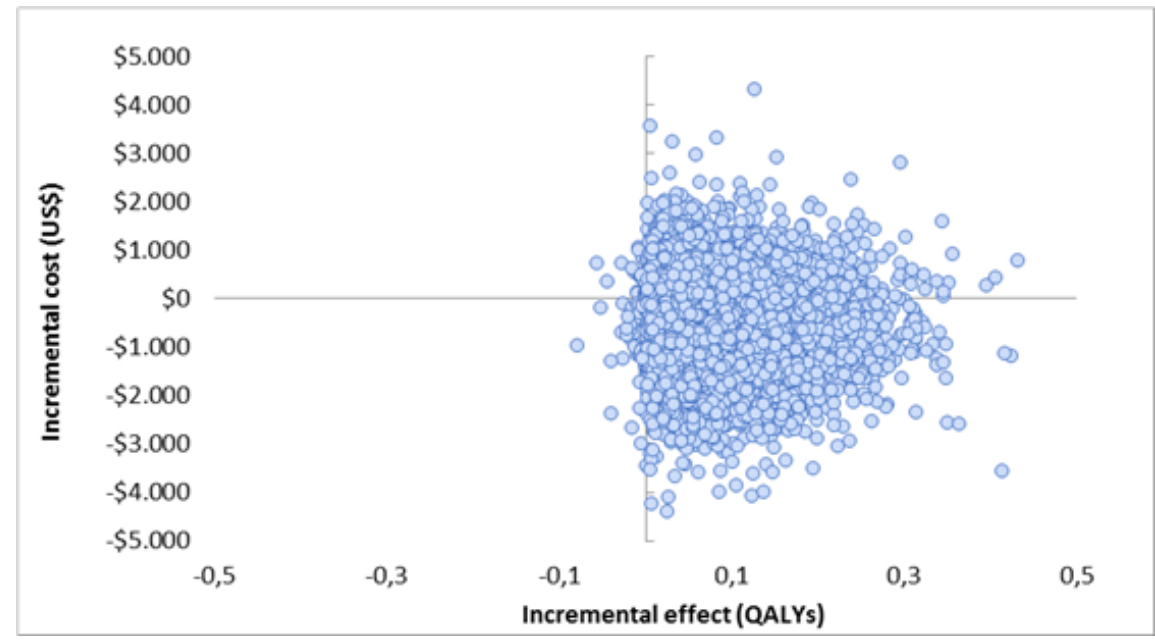

